\title{
Measuring cardiac strain using Laplacian smoothing splines
}

\author{
M. P. Griffin* F. Chen K. L. McMahon \\ G. Campbell S. J. Wilson S. E. Rose \\ M. Veidt ${ }^{\dagger}$ C. J. Bennett ${ }^{\ddagger}$ M. Wegner \\ D. M. Doddrell
}

(Received 21 September 2001)

\begin{abstract}
A scheme for obtaining strain maps from a set of MRI scans of the heart is presented. Using a technique known as the Spatial Modulation of Magnetization a set of MRI scans are generated with tag-lines distributed over the walls of the heart. As the heart moves, these tag-lines will move accordingly. Active contours or snakes are then used to extract the tag-lines from each MRI image, and the motion of the

*Center for Magnetic Resonance, University of Queensland, Brisbane, AustraliA. mailto:Mark.Griffin@cmr.uq.edu.au

${ }^{\dagger}$ Department of Mechanical Engineering, University of Queensland,

${ }^{\ddagger}$ Division of Medicine, Royal Brisbane Hospital, Brisbane, Australia.

${ }^{0}$ See http://anziamj.austms.org.au/V44/CTAC2001/Grif for this article, (C) Austral. Mathematical Soc. 2003. Published 1 April 2003. ISSN 1446-8735
\end{abstract} Brisbane, Australia. 
tag-lines determined. Laplacian smoothing splines are then used to estimate the displacement of points between the taglines. The strain experienced through out the heart is then a simple function of the associated displacement. Regional strain analysis is of vital importance in diagnosing different forms of heart disease, such as myocardial infarction or "heart attack". The method presented in this paper makes the analysis of cardiac strain feasible.

\section{Contents}

1 Introduction

2 The MRI scan

3 Processing the MRI scans

4 Generating a displacement surface using Laplacian splines

C256

5 Calculating strain from the displacement

C263

6 Results

C264

7 Conclusion

C266

References

C269

\section{Introduction}

According to the Australian Bureau of Statistics [1] 21.9\% of all deaths in 1998 were from ischaemic heart disease, making heart 
disease the most common cause of death, closely followed by cancer and stroke. It is therefore imperative to develop accurate diagnostic and research methods which characterise the mechanical properties of the heart and can be used to identify different cardiac conditions. The importance of the motion of the heart has been recognized since at least the 1600s [6]. Before the advent of medical imaging this consisted entirely of the observation of a patient's pulse. Techniques such as ultrasound and nuclear medicine were then used to measure a quantity known as the cardiac Ejection Fraction. This is simply a measure of the change in volume throughout the cardiac cycle:

$$
\text { Ejection Fraction }=\frac{V_{\max }-V_{\min }}{V_{\max }} .
$$

While these measures provide some global measure of the condition of the heart, it has been well recognised that the knowledge of the condition of different points throughout the heart would be invaluable for diagnosing different conditions. The use of radiopaque or ultrasonic markers was then considered $[2,5,13]$. These markers would be implanted on or in the myocardium during surgery, and these points could be traced using standard imaging techniques. However, there were a number of problems with these methods. In general there were insufficient markers to satisfactorily describe the geometry of the heart wall. The trauma of surgery and the presence of the markers also often altered the mechanical nature of the heart. Finally due to its invasive nature this approach was also only suitable for animal studies.

The current "gold standard" in cardiac diagnostics involves the measurement of regional strain through the use of MRI. Tag-lines are placed on the heart as part of the imaging experiment using a technique known as Spatial Modulation of Magnetization (see Figure 1). An overview of this MRI technique will be presented in Section 2. The motion of the heart is then determined from the 
displacement of these tag-lines throughout the cardiac cycle, and the corresponding strain can hence be determined.

This paper focuses on the data analysis required to convert a set of MRI images into a map of the regional strain. This analysis consists of the extraction of the contours of the heart wall and the tag-lines from a set of images, an interpolation method to generate a displacement surface from the displacement of the tag-lines, and the calculation of the strain maps from the displacement.

Some attempts have been made to automate the process of locating the heart wall contours and tag-lines, typically using some type of thresholding operation $[4,5,8]$. However, a number of problems with these techniques remain and in most cases an operator is required to trace these contours manually (as in our work).

Hermite interpolation is then typically used to generate the displacement surfaces $[8,10,12]$. Most of this work has focused on modelling the left ventricle where the geometry is significantly simpler, and is well described using prolate spheroid coordinates. To produce a method applicable to the more complex right ventricle geometry the work in this paper has been performed using standard Cartesian coordinates.

The paper is structured as follows. An overview of the MRI experiment is presented in Section 2, along with the SPAMM technique for placing the tag-lines. The use of snakes for extracting the tag-lines from a given set of images is discussed in Section 3. In Section 4, the generation of a displacement surface through the use of Laplacian splines. In Section 5, the generation of strain maps from the displacement surfaces is discussed. The paper concludes in Section 6 with some numerical results. 


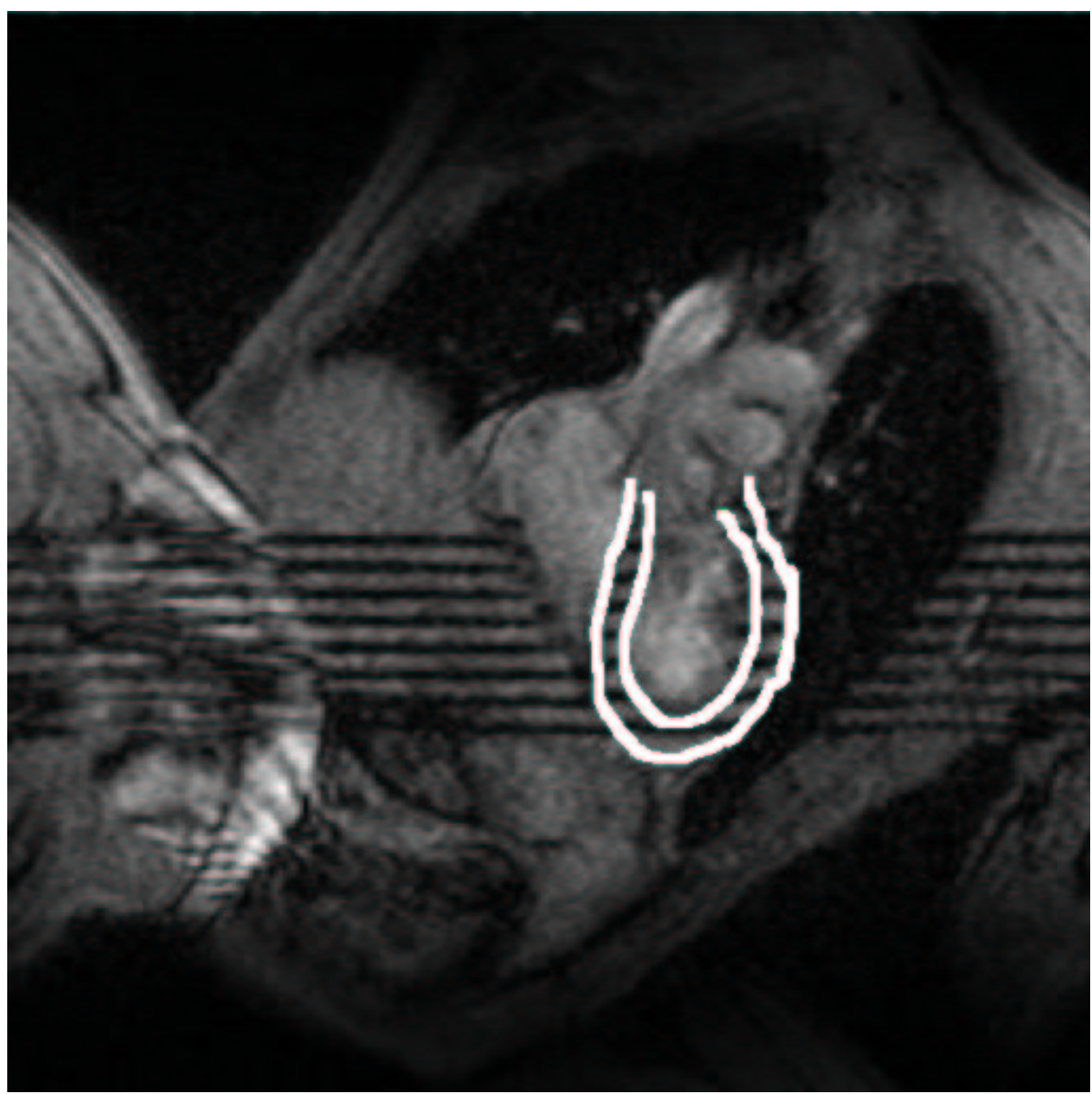

Figure 1: An MRI scan of the heart using Spatial Magnetization of Modulation (the contour of the heart is outlined). 


\section{The MRI scan}

A Magnetic Resonance Image (or MRI) is generated by placing a patient in a strong magnetic field (where a field gradient is used for spatial resolution). The net magnetization of the proton spins within the patient then align with the magnetic field. A second magnetic field is then pulsed which causes the net magnetization to rotate 90 degrees. The spin magnetization is then allowed to relax back to the equilibrium state, and the rate of relaxation at different points throughout the body is recorded and is presented as an MRI image.

Cardiac MRI relies heavily on the use of tag-lines generated through the Spatial Modulation of Magnetization [2]. In this case a pulse sequence of the second magnetic field causes the net magnetization over a regular pattern of lines to be reduced to zero prior to the imaging experiment. There is now no net magnetization to return to an equilibrium state, and hence no signal will be returned from these points. These altered spins will move throughout the cardiac cycle, hence the associated tag-lines are tracked to measure the motion of the heart (see Figure 1).

\section{Processing the MRI scans}

The first stage of the cardiac data analysis involves the extraction of the contours of the heart wall and the tag-lines from each image. Due to the inherent problems involved in automating the extraction of the heart contours, software was written allowing the user to trace the contours within each image. The border between the heart contours and the surrounding organs is often difficult to identify accurately, making the automation of such a task a significant problem. 
The extraction of the tag-lines was largely automated using a technique known as active contours or snakes. Snakes were introduced by Kass in 1988 [7]. Snakes consist of a set of points $V(s)=(x(s), y(s)), 0<s<1$, which are attracted towards a feature of interest within an image through minimising the energy term

$$
E_{\text {snake }}=E_{\text {internal }}+E_{\text {image }}+E_{\text {external }} .
$$

The internal energy describes the smoothness (that is, stretching and bending) of the points, and is defined as

$$
E_{\text {internal }}=\frac{1}{2} \int_{0}^{1} \alpha\left\|\frac{d v(s)}{d s}\right\|^{2}+\beta\left\|\frac{d^{2} v(s)}{d s^{2}}\right\|^{2} d s,
$$

where the coefficients $\alpha$ and $\beta$ control the amount of stretching and bending available.

The image energy is a function of the image intensity $(I)$ and its derivative with respect to the image axes $\left(i_{x}\right.$ and $\left.i_{y}\right)$ :

$$
E_{\text {image }}=\int_{0}^{1} \alpha I_{v(s)}+\beta\left(\frac{d I}{d x}\right)_{v(s)}+\gamma\left(\frac{d I}{d y}\right)_{v(s)} d s,
$$

where the coefficients $\alpha, \beta$ and $\gamma$ control the importance of the different intensity measures. Through minimising the image energy the points are attracted to the darker regions of an image and the regions where there is a large contrast in image intensity (that is, borders between light and dark regions).

Due to the nature of nonlinear optimisation, snakes may often be attracted to local minima or undesired features of interest. This is overcome by introducing a term labelled the external energy. In this case a user observes the snake moving towards the desired feature of interest, and stops the procedure when the snake appears to be moving in the wrong direction. The user then applies a point force 
which adjusts the snake points by an arbitrary amount back towards the desired feature of interest. In this way the snake is moved away from local minima and back towards the feature of interest within an image.

Figures 2 to 6 demonstrate the application of snakes to the taglines within a given image. The user starts by drawing the manually drawing the contours of the heart wall. Lines are then drawn which approximate the position of the tag-lines. These lines are converted into snakes which locate the tag-lines to the desired accuracy. Finally only those points which lie within the heart walls are recorded. In this way the tag-lines are located for each image throughout the set, and hence the displacement determined by considering the position of the tag-lines at different times.

\section{Generating a displacement surface using Laplacian splines}

Once the motion of the tag-lines has been determined, interpolation is used to estimate the motion across the heart wall. This work has attempted to outline the use of Laplacian splines [3] for this purpose, the most well-known of which is the thin plate spline. In the current application, the Laplacian splines are used to interpolate a set of $N$ data points where at a point $x_{i}$ in three-dimensional space a displacement of $y_{i}$ has been recorded along a direction $n\left(x_{i}\right)$. The spline $f$ (which defines a three-dimensional displacement for each point in space) can then be generated from this data by the minimization of

$$
\frac{1}{N} \sum_{i=1}^{N}\left(y_{i}-n\left(x_{i}\right) \cdot f\right)^{2}+\lambda \int_{\Re^{n}} \sum_{|\alpha|=1}^{m} \tau_{|\alpha|}\left(D^{\alpha} f\right)^{2},
$$




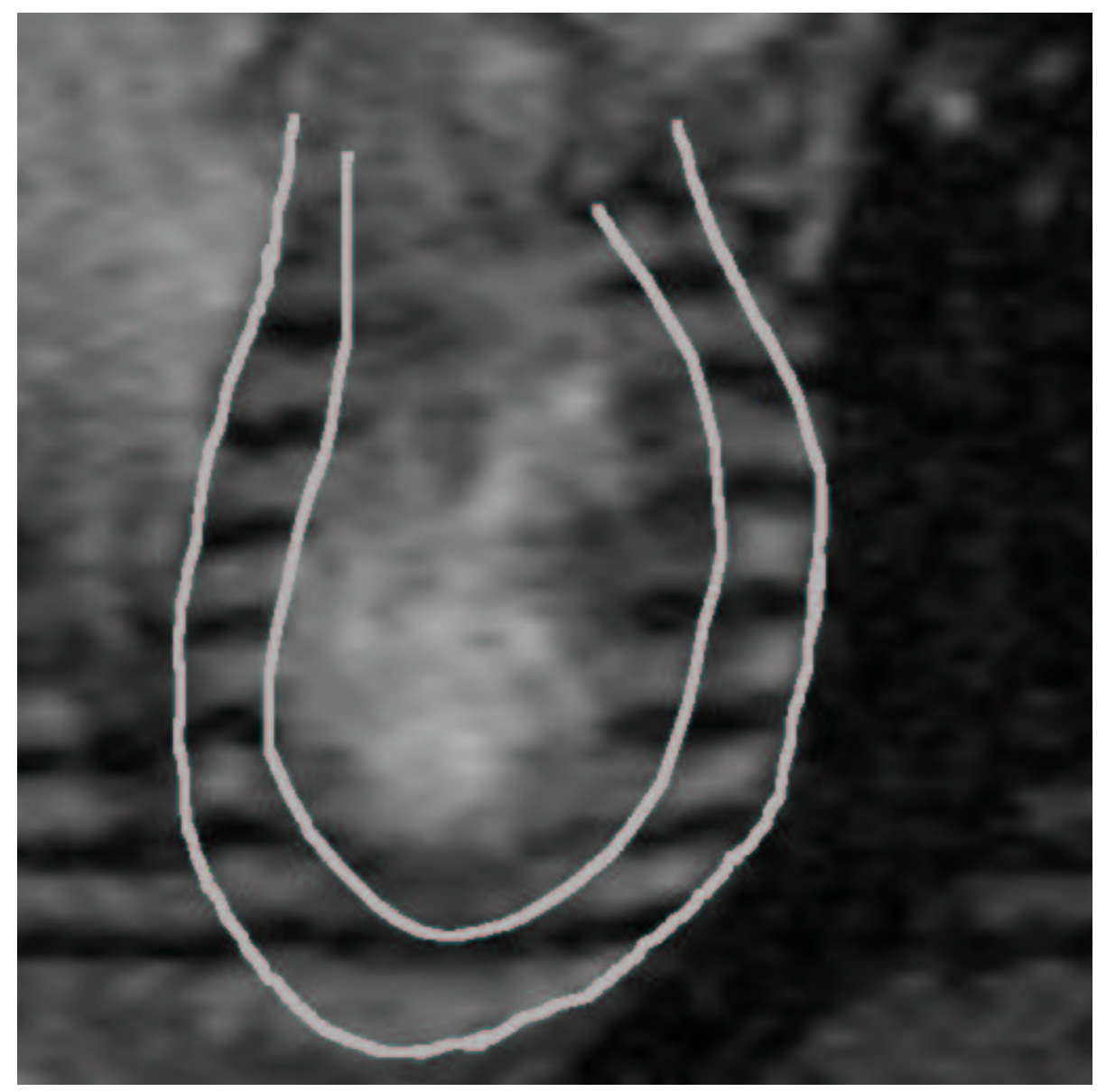

FIgURE 2: The user starts by manually drawing the contours of the heart wall. 


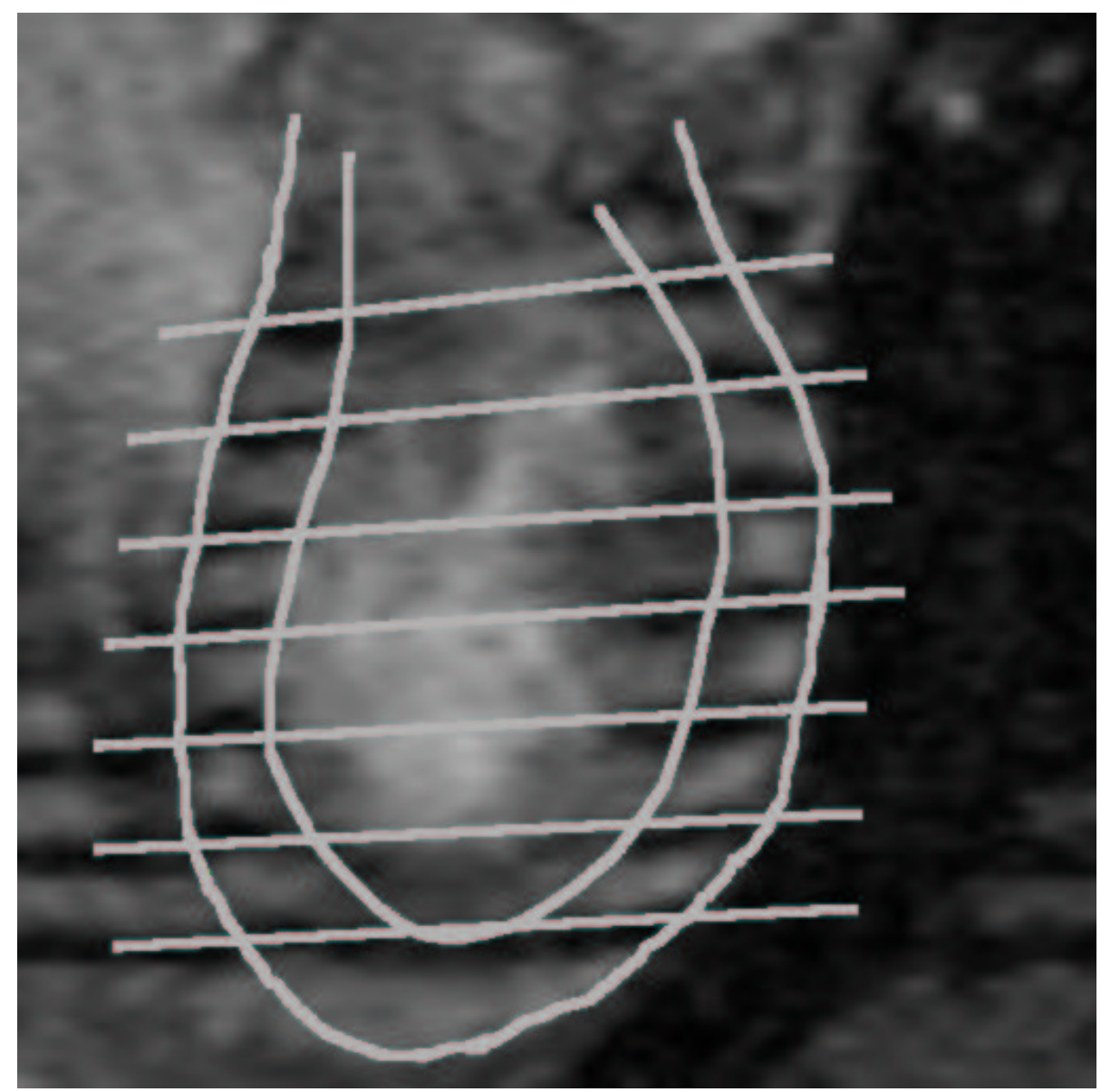

Figure 3: Lines are then drawn which approximate the position of the tag-lines. 


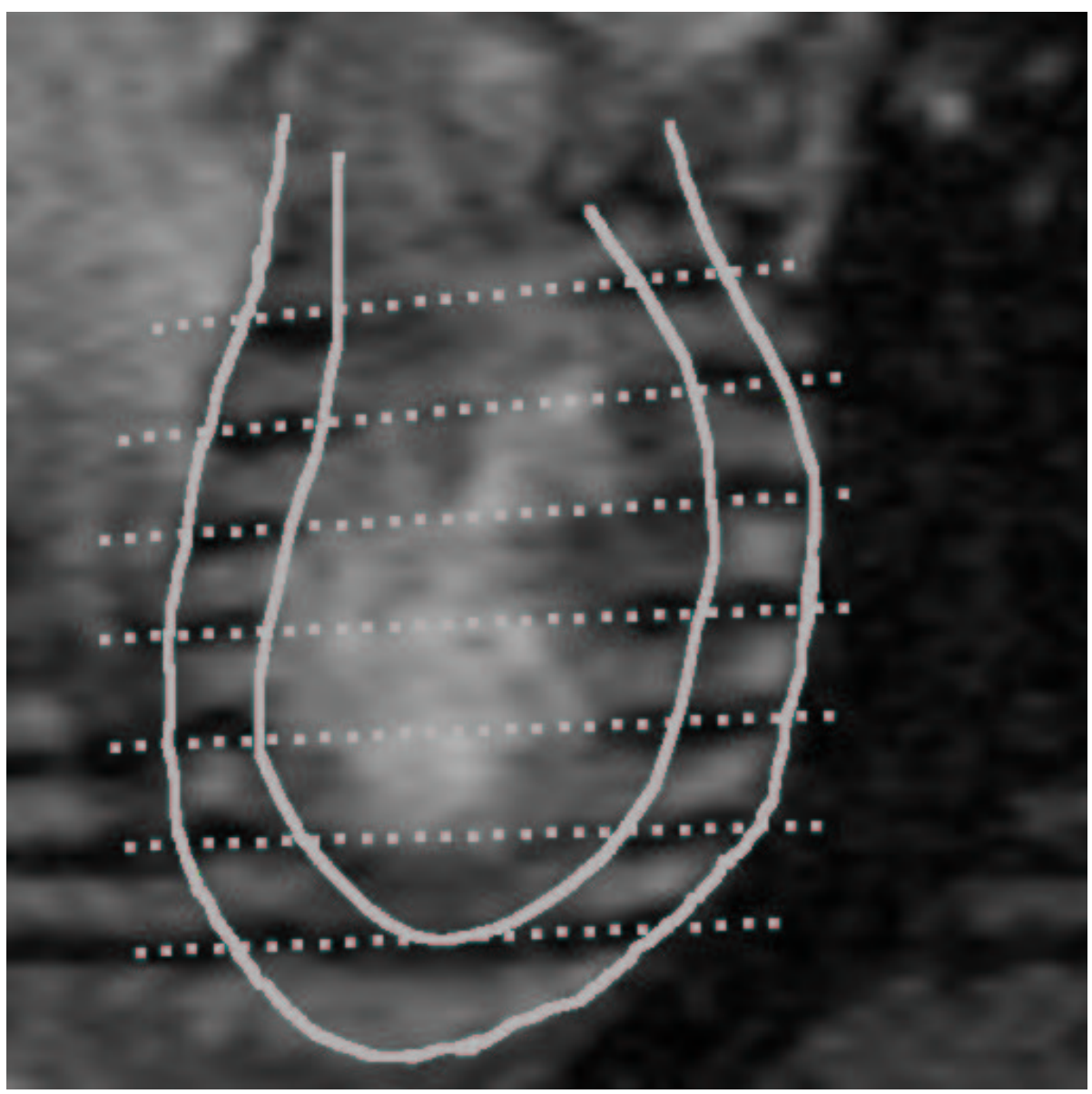

Figure 4: These lines are converted into a set of snakes. 


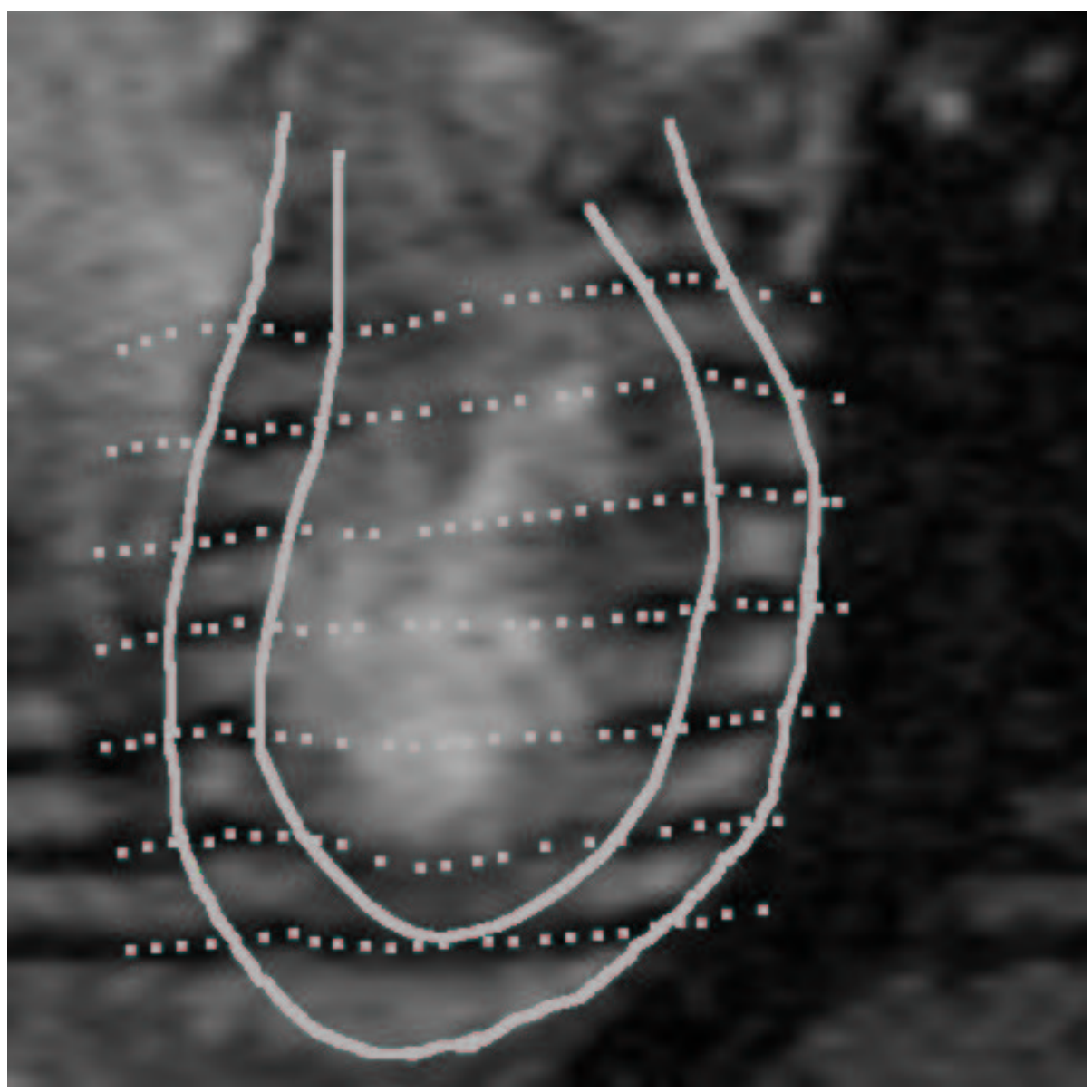

Figure 5: These snakes are attracted towards the tag-lines in an image. 


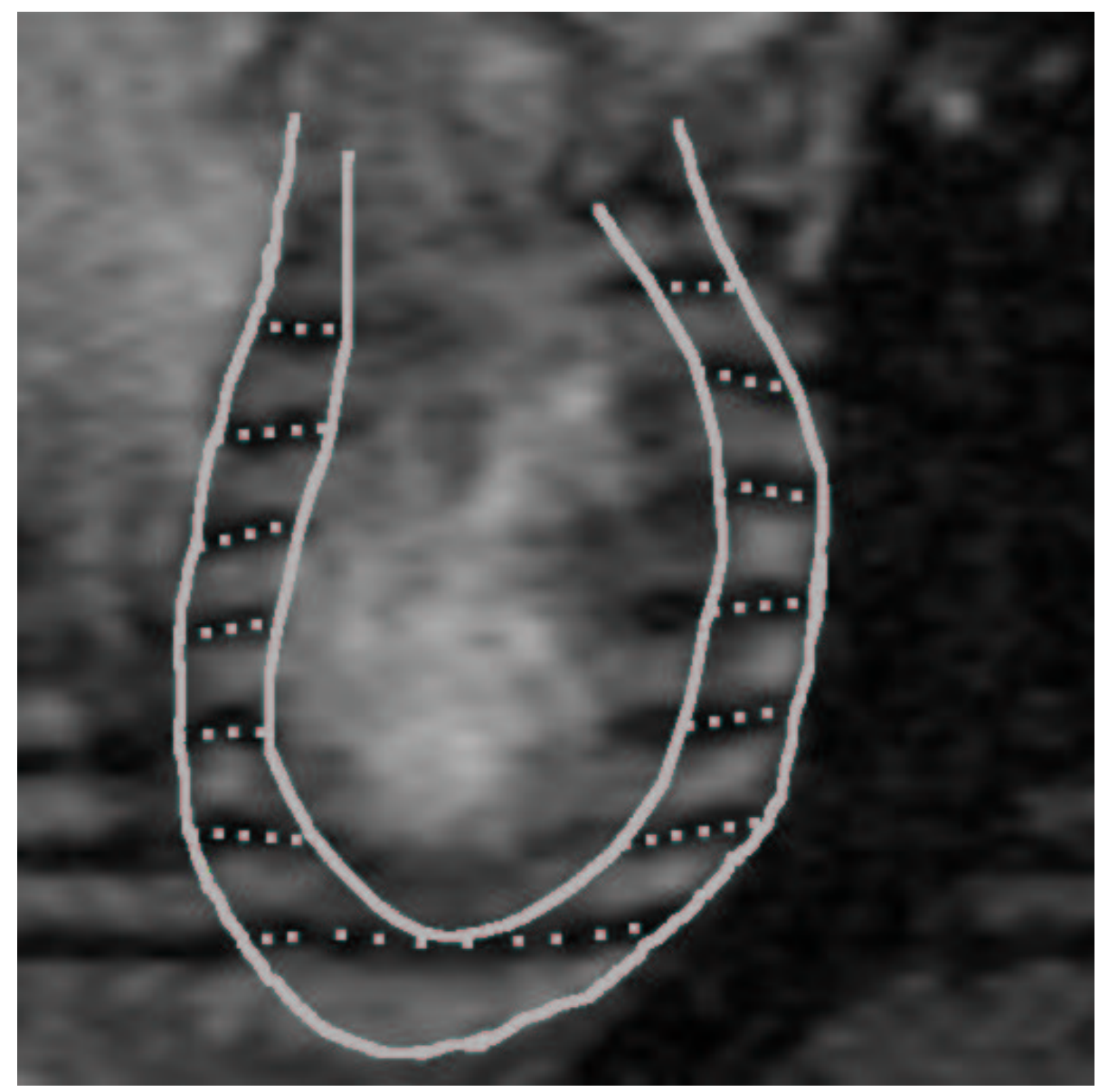

FIGURE 6: Finally only those points within the heart walls are considered. 
where the first term reflects how closely the spline fits the given data points and the second term reflects the smoothness of the spline according to a set of $m$ different derivatives of order $\alpha$. The smoothing parameter $\lambda$ reflects the relative importance between exactness and smoothness, and the importance of each derivative is given by the $\tau_{|\alpha|}$ coefficient. The minimization considered above results in

$$
f=\sum_{i=1}^{N} c_{i} K\left(\left|x-x_{i}\right|\right) n\left(x_{i}\right)+\sum_{j=1}^{M} d_{j} \Psi_{j} .
$$

The first term in this expression describes the contribution from each of the $N$ data points to the spline. The scalar function $K$ describes the influence of a data point at $x_{i}$ to a point $x$ on the spline. The precise form of these kernel functions is determined according to the spline derivatives employed to define the smoothness constraint [3]. For example, for a smoothness functional decomposed into the first and second order derivatives, the corresponding kernel is

$$
K_{12}(r)=\frac{1}{4 \pi \tau_{1} r}\left(1-\exp \left[-r \sqrt{\left(\tau_{1} / \tau_{2}\right)}\right]\right),
$$

where $r$ is the radius from $x_{i}$ to $x$, and $\tau_{1}$ and $\tau_{2}$ are user-defined parameters weighting the importance of the first and second derivatives. These analytical expressions commonly possess a singularity when $r$ equals zero. This is avoided by considering the limit in this region, so that for example

$$
K_{12}(r \rightarrow 0)=\frac{\sqrt{\left(\tau_{1} / \tau_{2}\right)}}{4 \pi \tau_{1}} .
$$

The contribution of a point $x_{i}$ to the spline describes a displacement in a direction $n\left(x_{i}\right)$ and is weighted by the scalar coefficient $c_{i}$. The $M$ basis functions $\Psi_{j}$ arise out of boundary conditions implicit in 
the minimization. These scalar functions are then weighted by coefficients along the three spatial directions according to the vector $d_{j}$. Given this form, a spline is fitted to a given set of data by determining the coefficients for the kernel and basis function $(c$ and $d$ ). These are found by minimising

$$
\frac{1}{N}\|y-S d-Q c\|^{2}+\lambda c^{T} Q c,
$$

where $S$ is an $N \times M$ matrix with entries $S_{i j}=n\left(x_{i}\right) \Psi_{j}$, and $Q$ is an $N \times N$ matrix with entries $Q_{i j}=n\left(x_{i}\right) K n\left(x_{j}\right)$. This minimization is expressed in standard linear algebra notation as

$$
\begin{aligned}
& (Q+\lambda N I) c+S d=y, \\
& S^{T} c=0 .
\end{aligned}
$$

For the work outlined in this paper, a numerical library known as Rkpack (available from Netlib [9]) was employed which contains procedures for the fitting of Laplacian splines. Surfaces describing the displacement along the $x, y$, and $z$ axes were generated by fitting Laplacian splines to the displacement of the tag-lines.

\section{Calculating strain from the displacement}

The generation of strain maps from the displacement surfaces is a fairly simple procedure. The nonlinear Lagrangian strain tensor $\epsilon[11]$ is defined as

$$
\epsilon_{i j}=\frac{1}{2}\left(\frac{\partial f_{i}}{\partial x_{j}}+\frac{\partial f_{j}}{\partial x_{i}}+\sum_{k=1}^{3} \frac{\partial^{2} f_{k}}{\partial x_{i} \partial x_{j}}\right),
$$


where $i, j=1,2,3$. Strain maps (such as those shown in Figure 7) can hence be generated.

\section{$6 \quad$ Results}

Strain maps of the heart are produced by generating MRI scans using Spatial Modulation of Magnetization, extracting the heart wall contours and tag-lines from the images, and fitting Laplacian splines to the displacement of the tag-lines.

To evaluate the accuracy of the interpolation a set of cardiac images was generated. A coordinate system was defined such that the length of the heart was aligned along the $z$-axis. Seven slices were then considered perpendicular to the $z$-axis and eight millimetres apart. For each slice four tagged images were generated, with the taglines (spaced six millimetres apart) lying along the $x, x+y$, $y$, and $x-y$ directions. Six slices were also considered intersecting along the $z$-axis and spaced thirty degrees apart. For these six slices the taglines were added perpendicular to the $z$-axis. For the single timeshot considered throughout the cardiac cycle, this resulted in a complete set of 34 images.

From these images, datapoints were then extracted one millimetre apart along the taglines, resulting in a dataset of 9754 points. The displacement at each point ranged between 0.000542 and 12.373 millimetres, with an average displacement of 3.762 millimetres.

Splines were then constructed from a subsample of 1626 points randomly selected from this dataset. A second subsample was constructed by adding noise to the displacement of thirty per cent of the original 1626 points. This noise had a Gaussian distribution with a standard deviation of 0.5 millimetres. The direction and position of 


\section{Strain}

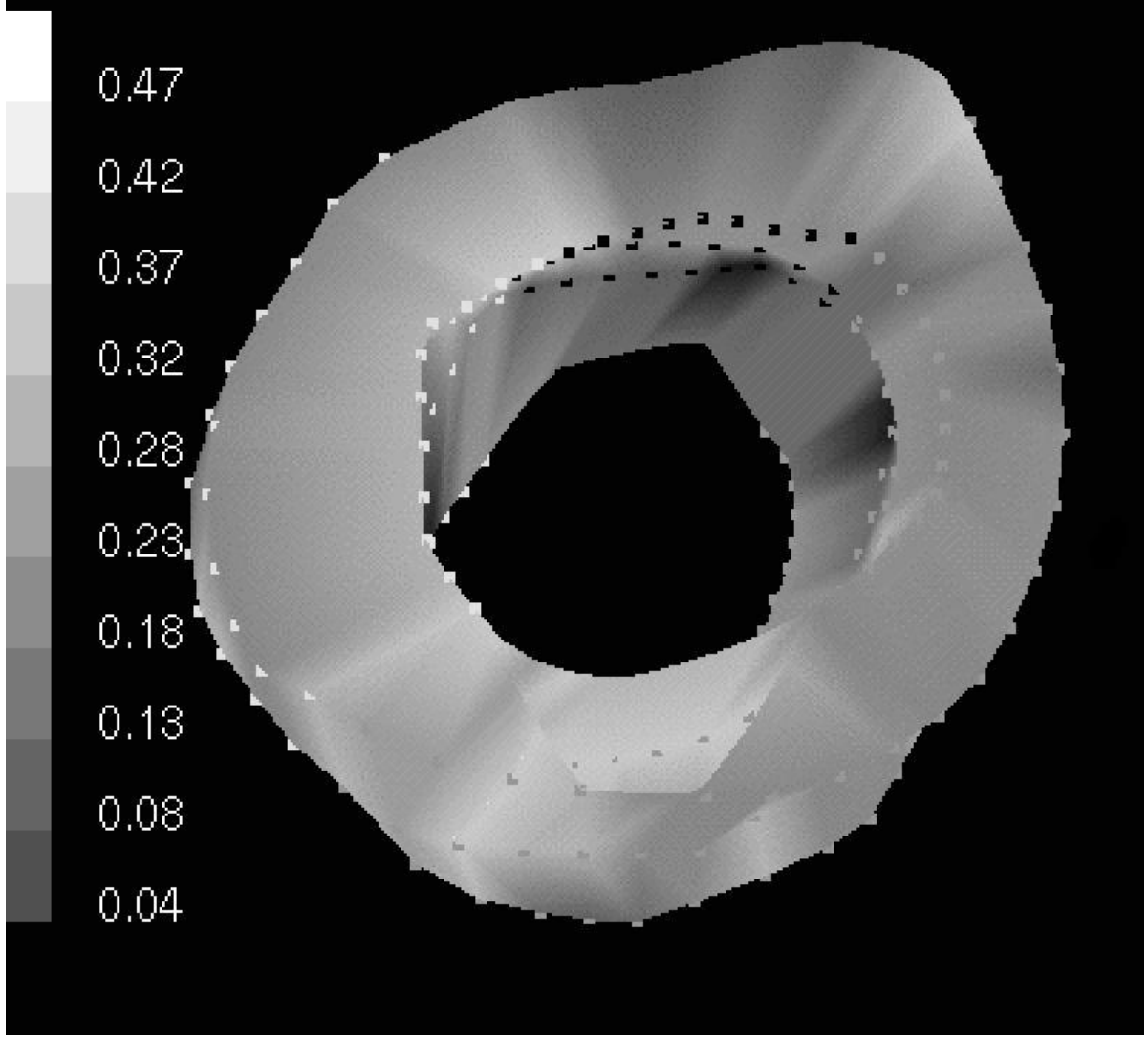

FiguRE 7: An example of the strain experienced by the heart. 
the data was not changed.

Laplacian splines were then constructed from these two datasets using a variety of different models according to the order of the derivatives employed in the smoothness constraints and the corresponding smoothness parameters $\tau$. The accuracy of these models was then determined (see Tables 1 and 2) by measuring the error between the experimental displacement measured at each of the original 9754 points and the displacements predicted by the splines.

In general the Laplacian splines contained a root mean squared error of less than half a millimetre, and tended to improve when higher derivatives were considered in the smoothness constraint. The addition of noise to the dataset resulted in a slight overall increase in error (of up to around ten per cent), yet the relative performance of the different models remains the same.

This interpolation procedure is one step in the generation of strain maps from a set of cardiac images. The time required for the interpolation is generally on the order of half an hour an SGI O2, and is easily outweighed by the highly user-intensive procedure for extracting the contours and tag-lines from the cardiac images. Techniques for automating this feature extraction (such as the incorporation of level set methodology) is currently being investigated.

\section{Conclusion}

This paper presents an overview of the data analysis required to generate strain maps from MRI scans of the heart. Tag-lines on the heart were added through an MRI technique known as Spatial Modulation of Magnetization. The heart wall contours and tag-lines 
TABLE 1: Maximum and root mean squared (RMS) error demonstrated by a range of Laplacian splines. Different splines were constructed by varying the order of the derivatives employed in the smoothness constraints and the corresponding smoothness parameters $\tau$. The splines were interpolated to a random selection of 1626 points out of a total set of 9754 points.

\begin{tabular}{|c|c|c|c|}
\hline Smoothness & Smoothness & Maximum & RMS \\
\hline Derivatives & Parameters & Error (mm) & Error $(\mathrm{mm})$ \\
\hline \multirow[t]{3}{*}{$1 \mathrm{st}+2 \mathrm{nd}$} & $0.99,0.01$ & 9.1562 & 2.1674 \\
\hline & $0.5,0.5$ & 5.3918 & 0.6749 \\
\hline & $0.01,0.99$ & 2.9061 & 0.2832 \\
\hline \multirow[t]{4}{*}{$1 \mathrm{st}+2 \mathrm{nd}+3 \mathrm{rd}$} & $0.98,0.01,0.01$ & 6.7766 & 1.0812 \\
\hline & $0.33,0.33,0.33$ & 4.3229 & 0.4635 \\
\hline & $0.01,0.98,0.01$ & 2.8976 & 0.2832 \\
\hline & $0.01,0.01,0.98$ & 2.5712 & 0.3137 \\
\hline \multirow[t]{3}{*}{$1 \mathrm{st}+3 \mathrm{rd}$} & $0.99,0.01$ & 6.8174 & 1.0964 \\
\hline & $0.5,0.5$ & 4.7480 & 0.5912 \\
\hline & $0.01,0.99$ & 2.5667 & 0.3146 \\
\hline $2 \mathrm{nd}$ & & 2.8647 & 0.2713 \\
\hline \multirow[t]{3}{*}{$2 \mathrm{nd}+3 \mathrm{rd}$} & $0.99,0.01$ & 2.8572 & 0.2716 \\
\hline & $0.5,0.5$ & 2.7020 & 0.2817 \\
\hline & $0.01,0.99$ & 2.4916 & 0.2998 \\
\hline $3 r d$ & & 2.4849 & 0.3007 \\
\hline \multirow[t]{3}{*}{$3 r d+4 t h$} & $0.99,0.01$ & 2.4839 & 0.3017 \\
\hline & $0.5,0.5$ & 2.7180 & 0.2560 \\
\hline & $0.01,0.99$ & 2.7047 & 0.2539 \\
\hline 4 th & & 2.6985 & 0.2542 \\
\hline 5 th & & 2.7686 & 0.2644 \\
\hline 6 th & & 2.7644 & 0.2672 \\
\hline
\end{tabular}


TABLE 2: Maximum and root mean squared (RMS) error demonstrated by a range of Laplacian splines. Different splines were constructed by varying the order of the derivatives employed in the smoothness constraints and the corresponding smoothness parameters $\tau$. The splines were interpolated to a random selection of 1626 points out of a total set of 9754 points. A Gaussian error with a standard deviation of $0.5 \mathrm{~mm}$ was added to thirty per cent of the 1626 points.

\begin{tabular}{|c|c|c|c|}
\hline Smoothness & Smoothness & Maximum & RMS \\
\hline Derivatives & Parameters & Error (mm) & Error (mm) \\
\hline \multirow[t]{3}{*}{$1 \mathrm{st}+2 \mathrm{nd}$} & $0.99,0.01$ & 9.1439 & 2.1680 \\
\hline & $0.5,0.5$ & 5.3826 & 0.6805 \\
\hline & $0.01,0.99$ & 3.0655 & 0.2995 \\
\hline \multirow[t]{4}{*}{$1 \mathrm{st}+2 \mathrm{nd}+3 \mathrm{rd}$} & $0.98,0.01,0.01$ & 6.7653 & 1.0842 \\
\hline & $0.33,0.33,0.33$ & 4.3253 & 0.4787 \\
\hline & $0.01,0.98,0.01$ & 3.0624 & 0.2992 \\
\hline & $0.01,0.01,0.98$ & 2.9384 & 0.3052 \\
\hline \multirow[t]{3}{*}{$1 \mathrm{st}+3 \mathrm{rd}$} & $0.99,0.01$ & 6.8061 & 1.0994 \\
\hline & $0.5,0.5$ & 4.7422 & 0.5323 \\
\hline & $0.01,0.99$ & 2.9354 & 0.3059 \\
\hline $2 n d$ & & 3.0333 & 0.2866 \\
\hline \multirow[t]{3}{*}{$2 \mathrm{nd}+3 \mathrm{rd}$} & $0.99,0.01$ & 3.0310 & 0.2865 \\
\hline & $0.5,0.5$ & 2.9873 & 0.2818 \\
\hline & $0.01,0.99$ & 2.9078 & 0.2684 \\
\hline $3 r d$ & & 2.9010 & 0.2677 \\
\hline \multirow[t]{3}{*}{$3 r d+4 t h$} & $0.99,0.01$ & 2.9008 & 0.2677 \\
\hline & $0.5,0.5$ & 2.8910 & 0.2674 \\
\hline & $0.01,0.99$ & 2.8666 & 0.2653 \\
\hline 4 th & & 2.8659 & 0.2660 \\
\hline 5 th & & 2.8774 & 0.2768 \\
\hline 6 th & & 2.9198 & 0.2899 \\
\hline
\end{tabular}


were extracted from the MRI images using manual means and a procedure known as active contours or snakes. Displacement surfaces were produced using Laplacian splines, and the subsequent strain maps were created.

\section{References}

[1] Australian Bureau of Statistics, http://www.abs.gov.au/ $\mathrm{C} 250$

[2] L. Axel and L. Dougherty. MR imaging of motion with Spatial Modulation of Magnetization. Radiology, 171:841-845, 1989. C251, C254

[3] P. Dierckx. Curve and surface fitting with splines. Clarendon Press, Oxford, 1995. C256, C262

[4] J. C. Fu, J. W. Chai and S. T. C. Wong. Wavelet-based enhancement for detection of left ventricular myocardial boundaries in magnetic resonance images. Magnetic Resonance Imaging, 18:1135-1141, 2000. C252

[5] I. Haber, D. N. Metaxas and L. Axel. Three-dimensional motion reconstruction and analysis of the right ventricle using tagged MRI. Medical Image Analysis, 4:335-355, 2000. $\mathrm{C} 251, \mathrm{C} 252$

[6] W. Harvey. Anatomical studies on the motion of the heart and blood in animals (1628) In R Willis trans, The works of William Harvey, pages 1-86. University of Pennsylvania Press, 1989. C251 
[7] M. Kass, A. Witkin and D. Terzopoulos. Snakes: Active contour models. International Journal of Computer Vision, 1:321-331, 1988. C255

[8] J. P. A. Kuijer, J. T. Marcus, M. J. W. Gotte, A. C. van Rossum and R. M. Heethaar. Three-dimensional myocardial strain analysis based on short and long-axis magnetic resonance tagged images using a 1D displacement field. Magnetic Resonance Imaging, 18:553-564, 2000. C252

[9] Netlib Repository, http://www.netlib.org/ C263

[10] P. M. F. Nielsen, I. J. Le Grice, B. H. Smaill and P. J. Hunter. Mathematical model of geometry and fibrous structure of the heart. American Journal of Radiology, 260:H1365-H1378, 1991. C252

[11] R. J. Roark. Formulas for stress and strain. McGraw-Hill, New York, 1965. C263

[12] A. A. Young and L. Axel. Three-dimensional motion and deformation of the heart wall : estimation with Spatial Modulation of Magnetization. Radiology, 185:241-247, 1992. $\mathrm{C} 252$

[13] A. A. Young, P. J. Hunter and B. H. Smaill. Epicardial surface estimation from coronary angiograms. Computer Vision, Graphics, and Image Processing, 47:111-127, 1989. $\mathrm{C} 251$ 